\title{
POSITIVITY OF SPIN FOAM AMPLITUDES
}

\author{
JOHN C. BAEZ AND J. DANIEL CHRISTENSEN
}

\begin{abstract}
The amplitude for a spin foam in the Barrett-Crane model of Riemannian quantum gravity is given as a product over its vertices, edges and faces, with one factor of the Riemannian $10 j$ symbols appearing for each vertex, and simpler factors for the edges and faces. We prove that these amplitudes are always nonnegative for closed spin foams. As a corollary, all open spin foams going between a fixed pair of spin networks have real amplitudes of the same sign. This means one can use the Metropolis algorithm to compute expectation values of observables in the Riemannian Barrett-Crane model, as in statistical mechanics, even though this theory is based on a real-time $\left(e^{i S}\right)$ rather than imaginary-time $\left(e^{-S}\right)$ path integral. Our proof uses the fact that when the Riemannian $10 j$ symbols are nonzero, their sign is positive or negative depending on whether the sum of the ten spins is an integer or half-integer. For the product of $10 j$ symbols appearing in the amplitude for a closed spin foam, these signs cancel. We conclude with some numerical evidence suggesting that the Lorentzian $10 j$ symbols are always nonnegative, which would imply similar results for the Lorentzian Barrett-Crane model.
\end{abstract}

\section{INTRODUCTION}

Physicists have long found it difficult to deal with the oscillatory behavior of the integrand in real-time Feynman path integrals of the form

$$
\int f(x) e^{i S(x)} \mathcal{D} x \text {. }
$$

For this reason, we have become accustomed to using Wick rotation to switch to imaginary time, thus replacing these integrals by those of the form

$$
\int f(x) e^{-S(x)} \mathcal{D} x
$$

where the positivity of the exponential allows one to use concepts from probability theory. This is helpful not only in analytical work but also in numerical computations. For example, computations in lattice field theory rely heavily on techniques such as the Metropolis algorithm. They give powerful tools for computing the latter sort of integral, but are typically useless for those of the former sort.

Unfortunately, the strategy of Wick rotation becomes problematic in the context of quantum gravity. The basic difficulty is that in the absence of a favored time coordinate, the replacement $t \mapsto i t$ makes no sense unless one can prove that the results obtained thereby are independent of the choice of the $t$ coordinate. A great deal of work on 'Euclidean quantum gravity' is of dubious relevance to real-world physics because of inattention to this issue.

Luckily, in the absence of topology change (i.e. for spacetimes of the topology $\mathbb{R} \times S$ ) there exist theorems justifying Wick rotation for certain diffeomorphism-invariant field theories [3]. Taking the prohibition against topology change seriously has led to models

Date: February 21, 2002. 
of quantum gravity in which Wick rotation is indeed justified, and these appear to be better-behaved than those previously considered in Euclidean quantum gravity [1], 2].

Nonetheless, there are still reasons to be interested in real-time Feynman path integrals in quantum gravity. After all, the prohibition against topology change might be a limitation of the Wick rotation method rather than an actual law of physics. Moreover, the original Feynman integrals have a certain conceptual priority over their Wick-rotated counterparts: quantum theory is ultimately about amplitudes, not probabilities.

Yet another reason is that there are some very interesting models of quantum gravity based on real-time path integrals: namely, spin foam models [5, 6, 10, 11, 20, 22, 23]. In a spin foam model of quantum gravity, the real-time path integral is rewritten as a sum over 'spin foams': 2-dimensional cell complexes with faces labelled by group representations and edges labelled by intertwiners. This sum has been rigorously shown to converge in some of these models 14, 21, at least for a fixed triangulation of spacetime. The next step is to determine whether these models have good behavior at large distance scales: that is, whether they reduce to general relativity in a suitable limit. For this, we need to compute expectation values of observables, e.g. by numerical methods.

Here we show that for a specific model of this sort - the Riemannian Barrett-Crane model - one can do such computations using the Metropolis algorithm, because the amplitudes for closed spin foams are nonnegative. This model is not physically realistic: it is a version of 'Riemannian quantum gravity', that is, the quantum field theory based on a real-time path integral involving the Einstein-Hilbert action for Riemannian metrics. Since the Cauchy problem is ill-posed in classical Riemannian general relativity, we should expect to run into problems with the quantum theory at some point. Nonetheless, the Riemannian Barrett-Crane model has so far proved to be a useful guide to work on the physically more important but mathematically more challenging Lorentzian Barrett-Crane model. In particular, we conjecture that the nonnegativity of spin foam amplitudes holds for the Lorentzian model as well as the Riemannian one.

In Section 2 we prove the nonnegativity of spin foam amplitudes in two versions of the Riemannian Barrett-Crane model: the version due to De Pietri, Freidel, Krasnov and Rovelli [15], and the version with modified edge and face amplitudes due to Perez and Rovelli 222. In the Perez-Rovelli version the partition function has been shown to converge 21; in the DFKR version the partition function appears to diverge [8]. However, in both versions the spin foam vertex amplitudes are given by the Riemannian $10 j$ symbols, and the key fact we need to show is that the product of these $10 j$ symbols over all vertices of a closed spin foam is nonnegative. We prove this in two steps. In Lemma 1, we show that when the Riemannian $10 j$ symbol is nonzero, its sign is positive or negative depending on whether the sum of the ten spins in question is an integer or half-integer. In Lemma 5 , we show that all the resulting minus signs cancel when we take the product of $10 j$ symbols over all vertices of a closed spin foam. This immediately gives the desired result. As a corollary of this result, we show that all open spin foams going between fixed spin networks have real amplitudes of the same sign. Finally, we present some numerical evidence supporting our conjecture that the Lorentzian $10 j$ symbols are always nonnegative. 


\section{The Riemannian Model}

In what follows we shall freely make use of standard graphical notation for $\mathrm{SU}(2)$ spin networks [13, 17]. We normalize trivalent vertices so that

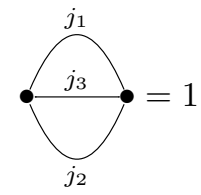

whenever the spins $j_{1}, j_{2}, j_{3}$ form an admissible triple: that is, satisfy the triangle inequality and sum to an integer. (When the spins do not form an admissible triple, we define the trivalent vertex to be zero.) As this normalization sometimes requires dividing by the square root of a negative number, it introduces a potential sign ambiguity. Luckily, in our calculations trivalent vertices always come in matching pairs, so these signs cancel, leaving an unambiguous result. This is important, because signs are of the essence in all to come.

We shall also use the standard notation for simple spin networks [10, 26]. In such a spin network, labelling an edge by the spin $j$ really means that it is labelled by irreducible representation $j \otimes j$ of the group $\operatorname{Spin}(4)=\mathrm{SU}(2) \times \mathrm{SU}(2)$. Such representations are called 'simple', and they all descend to representations of $\mathrm{SO}(4)$. Unlike $\mathrm{SU}(2)$ spin networks, which change sign when one inserts a twist in the framing of any edge labelled by a half-integer spin, simple spin networks are framing-independent. In a simple spin network, any unlabelled 4-valent vertex is really labelled by the Barrett-Crane intertwiner. This intertwiner is defined in terms of $\mathrm{SU}(2)$ intertwiners by:
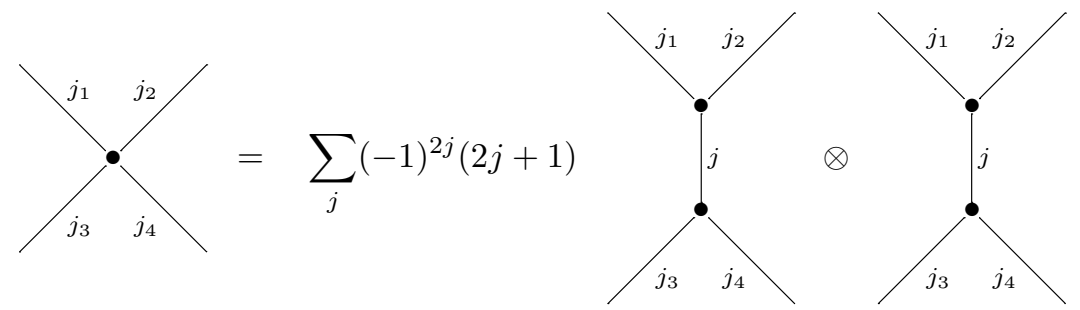

where we sum over spins $j$ such that the triples $j_{1}, j_{2}, j$ and $j_{3}, j_{4}, j$ are both admissible. Superficially, this definition involves splitting the four edges incident to the vertex into a pair of 'incoming' edges and a pair of 'outgoing' ones. However, the Barrett-Crane intertwiner is actually independent of this choice. For example, we also have
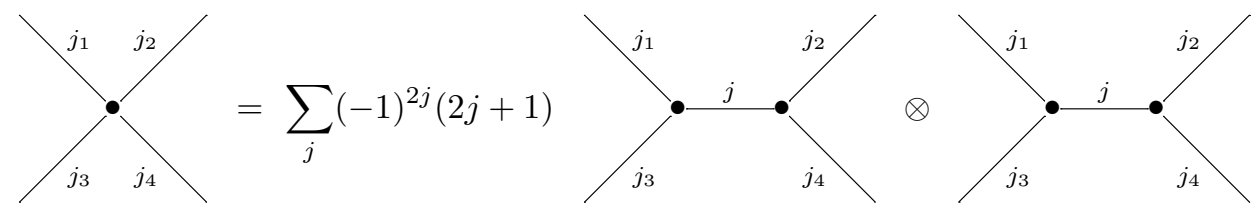

This fact is stated in Barrett and Crane's original paper [10]; a formal proof was given by Yetter [26].

It will be technically useful to modify the Barrett-Crane intertwiner by removing the signs from the above formulas. Our final results will concern the original Barrett-Crane intertwiner, but our intermediate calculations will use the intertwiner without signs. In 
this modified intertwiner we use a dotted line to indicate the splitting of incident edges into 'incoming' and 'outgoing' pairs:
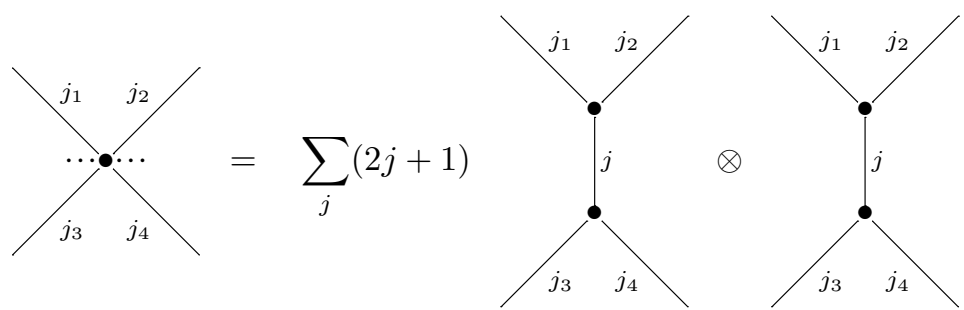

In fact, this modified intertwiner only differs from the usual one by an overall sign.

\section{Lemma 1.}

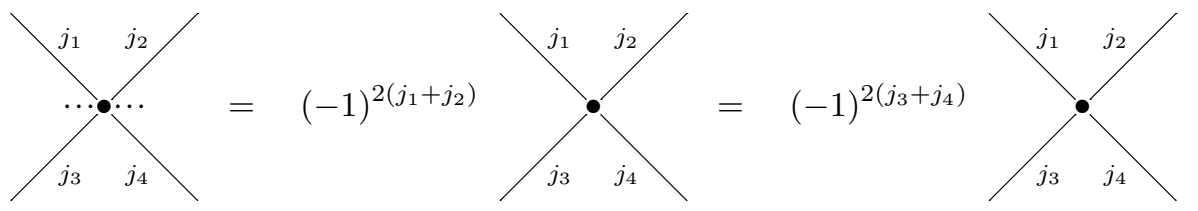

It follows that

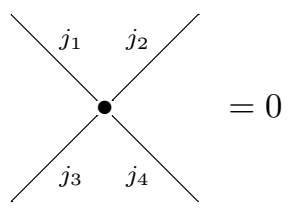

unless $j_{1}+\cdots+j_{4}$ is an integer.

Proof - Since the triples $j_{1}, j_{2}, j$ and $j_{3}, j_{4}, j$ are admissible in the sum

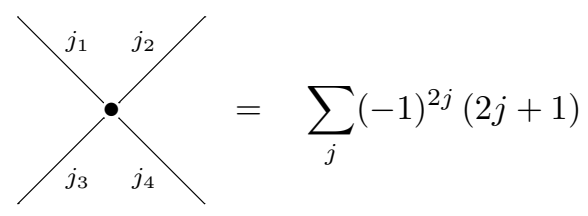

we have $(-1)^{2 j}=(-1)^{2\left(j_{1}+j_{2}\right)}=(-1)^{2\left(j_{3}+j_{4}\right)}$, which yields the equations to be proved.

This means that the modified intertwiner changes at most by a sign when we change the splitting. For example, consider
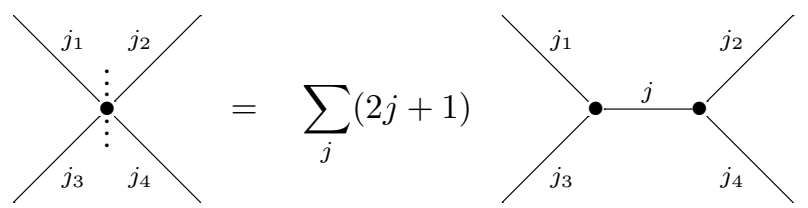

$\otimes$

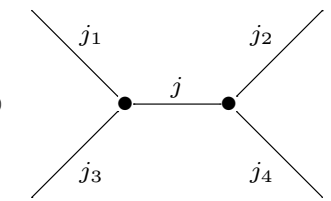

This satisfies: 


\section{Lemma 2.}

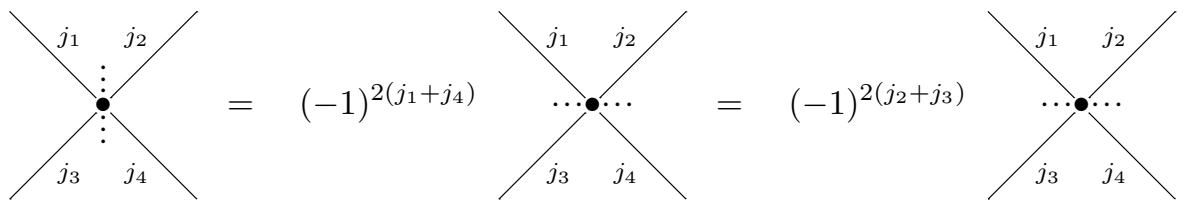

Proof - By Lemma 11 we have

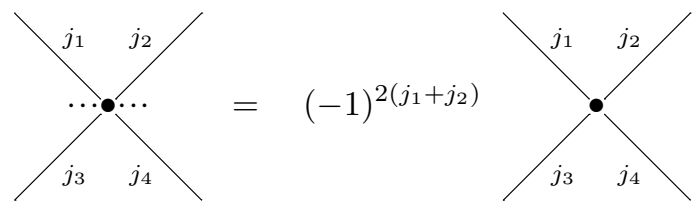

Rotating the pictures clockwise and relabelling, we have

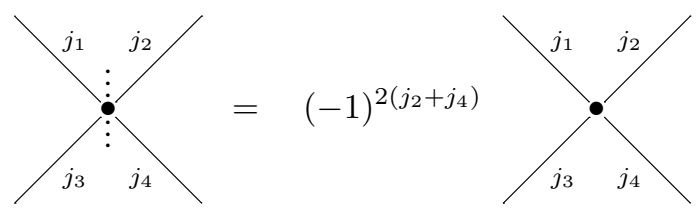

Combining these equations, it follows that

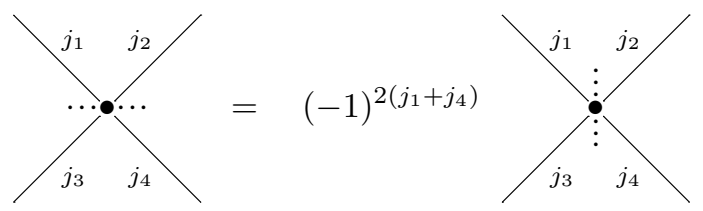

as desired. A similar argument where we rotate counterclockwise gives

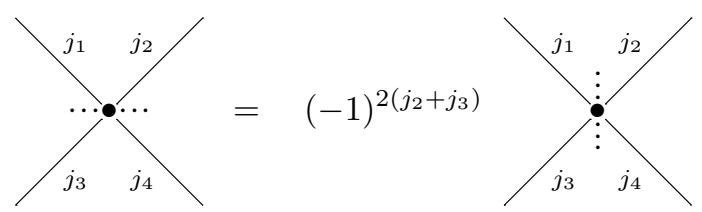

The usual Riemannian $10 j$ symbol [10] is the function of 10 spins obtained by evaluating the following closed $\mathrm{SU}(2) \times \mathrm{SU}(2)$ spin network:

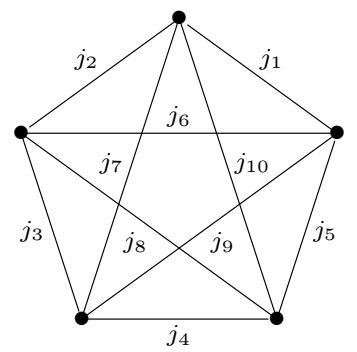


where the vertices are labelled by Barrett-Crane intertwiners. Mimicking this construction, we can also define a modified $10 j$ symbol using the modified Barrett-Crane intertwiners:

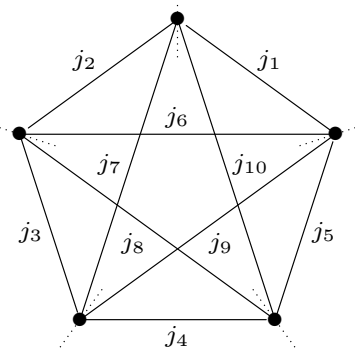

Here the choice of splittings really matters: by Lemma 2, if we change the splitting at any vertex, the value of the spin network might change sign. The nice thing about the above choice is that it always gives a nonnegative result:

\section{Lemma 3.}

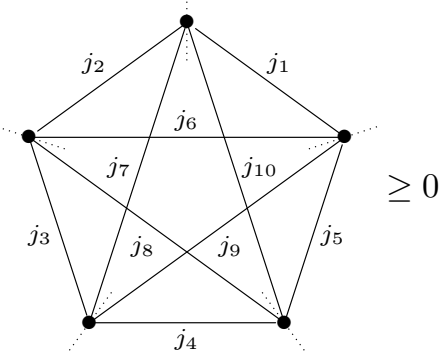

Proof - Using the definition of the modified Barrett-Crane intertwiner, the modified $10 j$ symbol above can be rewritten as the following weighted sum of $\mathrm{SU}(2)$ spin networks:

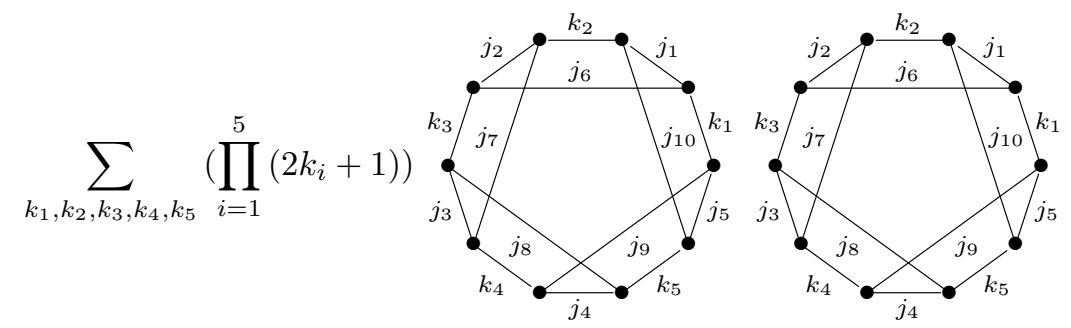

Since the value of a disjoint union of spin networks is the product of their values, it follows that

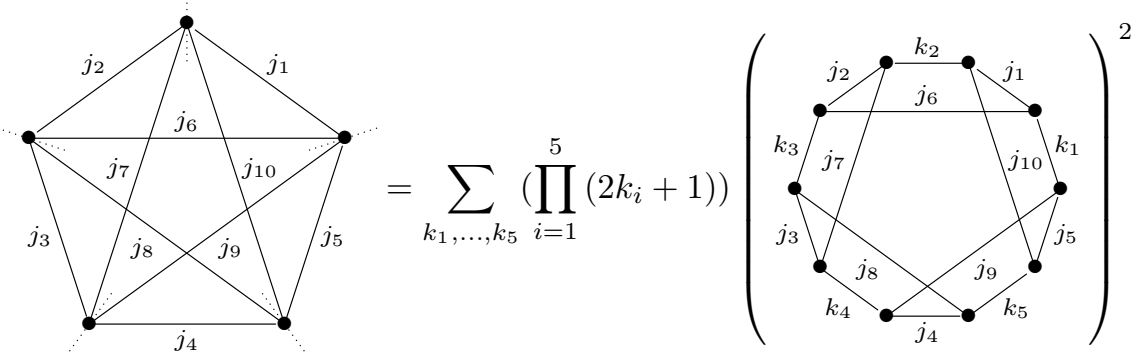


To show that the modified $10 j$ symbols are positive, it thus suffices to show that this $\mathrm{SU}(2)$ spin network, called a $15 j$ symbol:

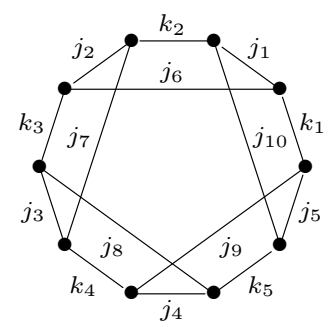

evaluates to a real number.

To see this, first note that the only place where complex numbers are essential to the theory of trivalent $\mathrm{SU}(2)$ spin networks is in the normalization of the trivalent vertex. If we used the projection from the tensor product $j_{1} \otimes j_{2}$ to the summand $j_{3}$ as our trivalent vertex whenever $j_{1}, j_{2}, j_{3}$ is an admissible triple, all closed trivalent $\mathrm{SU}(2)$ spin networks would evaluate to real numbers, since we could define them using the representation theory of $\mathrm{SL}(2, \mathbb{R})$ instead of $\mathrm{SU}(2)$. However, this would not give

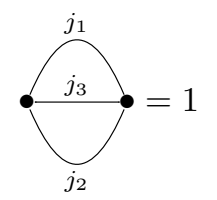

Writing $\theta\left(j_{1}, j_{2}, j_{3}\right)$ for the unnormalized evaluation of this network, we see that to achieve this normalization we must divide the projection $p: j_{1} \otimes j_{2} \rightarrow j_{3}$ by the square root of $\theta\left(j_{1}, j_{2}, j_{3}\right)$. The formula in Section 9.10 of the book by Kauffman and Lins [17] makes it obvious that $\theta\left(j_{1}, j_{2}, j_{3}\right)$ is positive (resp. negative) when $j_{1}+j_{2}+j_{3}$ is even (resp. odd). This means that when we use normalized trivalent vertices, a closed trivalent $\mathrm{SU}(2)$ spin network evaluates to a real number times the product over all vertices $v$ of $i^{j_{1}+j_{2}+j_{3}}$, where $j_{1}, j_{2}, j_{3}$ are the spins labelling the three edges incident to $v$. Since the spin label for each edge shows up twice in this product, it follows that the spin network evaluates to a real number times $i^{2 J}$, where $J$ is sum of the spin labels of all edges.

It therefore suffices to show that whenever the $15 j$ symbol is nonzero, this quantity $J$ is an integer. To do this, choose any vertex in the $15 j$ symbol spin network and consider its images $v_{1}, \ldots, v_{5}$ under the action of $\mathbb{Z}_{5}$ as rotations of the picture. Each edge is incident to exactly one of the vertices $v_{i}$, so $J$ is the sum over $1 \leq i \leq 5$ of the sum of spins labelling the edges incident to $v_{i}$. However, a trivalent vertex can only be nonzero if the sum of spins incident to it is an integer. It follows that for the $15 j$ symbol to be nonzero, $J$ must be an integer.

The fact we use here about the $15 j$ symbols has a nice generalization: any trivalent $\mathrm{SU}(2)$ spin network evaluates to real number if its underlying graph has no loops containing an odd number of edges. The reason is that in a nonzero $\mathrm{SU}(2)$ spin network, the edges with non-integral labels can always be partitioned into disjoint loops. If these loops all have an even number of edges, the total of all of the spins must be an integer, so the above argument shows the spin network evaluates to a real number.

Using Lemma 3, we can easily determine the sign of the usual $10 j$ symbol: 


\section{Lemma 4.}
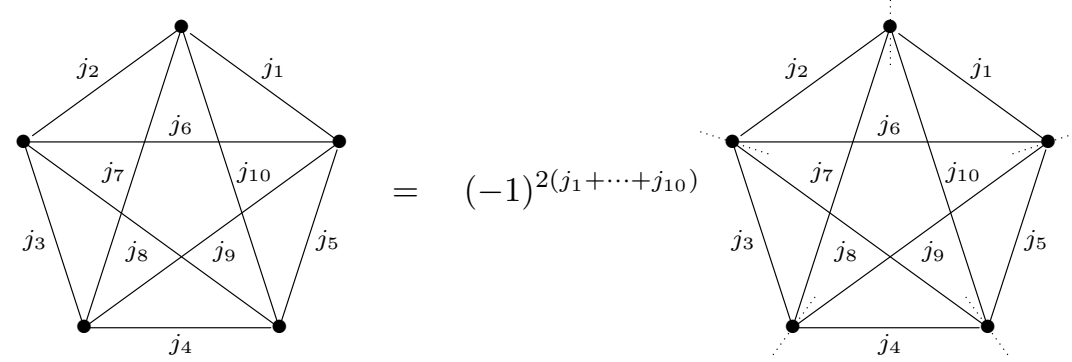

It follows that when

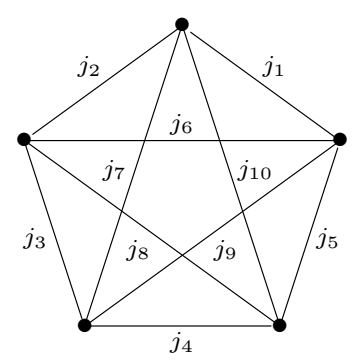

is nonzero, it is positive (resp. negative) when $j_{1}+\cdots+j_{10}$ is an integer (resp. halfinteger).

Proof - If we go around this graph counterclockwise:

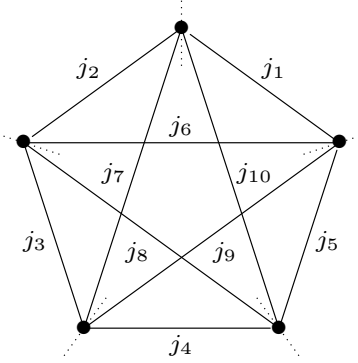

each of the ten edges is incoming to exactly one of the modified Barrett-Crane intertwiners. By Lemma 1, it follows that
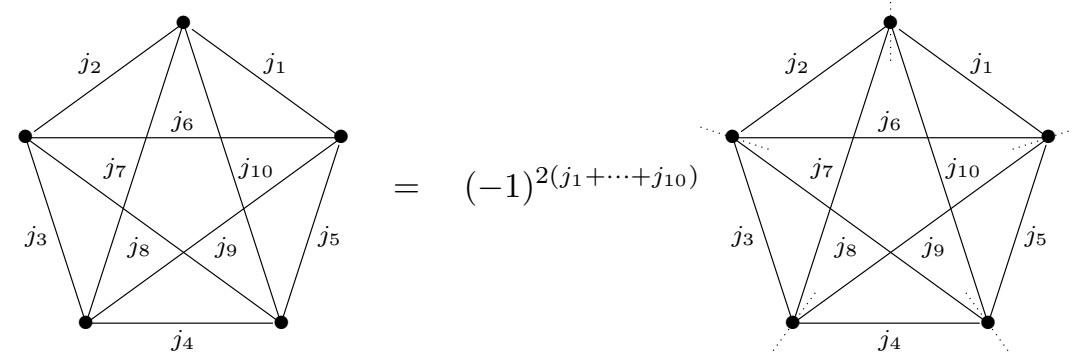

The rest follows from Lemma 3 . 
Finally, we show that these signs cancel when we evaluate a closed spin foam of the sort that appears in the Barrett-Crane model. Here we assume familiarity with the basic definitions concerning piecewise-linear CW complexes and their homology [5, 18, 24].

Lemma 5. Let $K$ be any 2-dimensional piecewise-linear $C W$ complex, and let $c$ be a 2-cycle on $K$ with $\mathbb{Z}_{2}$ coefficients. Let $c(f)$ denote the value of $c$ on any face (i.e., 2-cell) $f$, and let $n(f)$ denote the number of vertices of the face $f$ (which equals the number of its edges). Then $\sum_{f} n(f) c(f)=0$.

Proof - Write $f>e$ when the face $f$ has the $e$ as one of its edges, and let $(\partial c)(e)$ be the boundary chain of $c$ evaluated at the edge $e$. Then

$$
\sum_{f} n(f) c(f)=\sum_{e} \sum_{f>e} c(f)=\sum_{e}(\partial c)(e)=\sum_{e} 0=0
$$

We now reach our main result. Here, and in the corollary, we are using the original $10 j$ symbols, so this result is independent of any choices of splittings of vertices.

Theorem 1. Let $F$ be a closed spin foam whose underlying 2-dimensional piecewiselinear $C W$ complex $K$ is the dual 2-skeleton of simplicial complex formed by taking a finite set of 4-simplices and gluing them pairwise along all their tetrahedral faces. Then $\prod_{v} A_{v} \geq 0$, where $v$ runs over the vertices of $K$ and $A_{v}$ is the $10 j$ symbol associated to $v$.

Proof - We assume that the product $\prod_{v} A_{v}$ is nonzero, and show that the product of the signs from Lemma 1 is 1.

Since the product is nonzero, each $10 j$ symbol is nonzero, and therefore each BarrettCrane intertwiner is nonzero. For each edge in the spin foam (corresponding to a 3simplex in the simplicial complex) there are exactly four faces having it in their boundary (corresponding to the four triangles in the 3 -simplex). Because the associated BarrettCrane intertwiner is nonzero, Lemma 1 shows that the sum of the spins on these four faces is an integer. From the spin foam $F$ we construct a 2-chain $c$ with values in the multiplicative group $\{ \pm 1\}$ by setting $c(f)=(-1)^{2 j_{f}}$, where $j_{f}$ is the spin labelling the face $f$. The condition above says that $c$ is a cycle. Translating Lemma 5 into

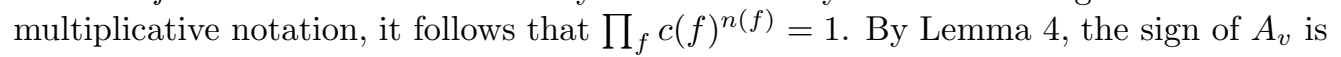
$\prod_{f>v}(-1)^{2 j_{f}}=\prod_{f>v} c(f)$, where $f$ runs over all the 2 -cells having $v$ as a vertex. The sign of $\prod_{v} A_{v}$ is therefore $\prod_{v} \prod_{f>v} c(f)=\prod_{f} c(f)^{n(f)}=1$.

In addition to a product of $10 j$ symbols, one for every vertex, the Barrett-Crane amplitude for a spin foam also involves a product of factors coming from spin foam edges and faces. The precise formulas for these factors are somewhat controversial: Perez and Rovelli [22] have proposed a modification of the original formulas given by Barrett and Crane [10]. However, in both versions of the Barrett-Crane model, these factors are nonnegative. It follows from the above theorem that in both the original Barrett-Crane model and the Perez-Rovelli version, the amplitude for any closed spin foam is nonnegative.

We can generalize this theorem to open spin foams as follows:

Corollary 1. Let $F: \psi \rightarrow \psi^{\prime}$ be an open spin foam whose underlying 2-dimensional piecewise-linear $C W$ complex is the dual 2-skeleton of a simplicial complex formed by taking a finite set of 4-simplices and gluing them pairwise along all their tetrahedral 
JOHN C. BAEZ AND J. DANIEL CHRISTENSEN

faces except those in which the spin networks $\psi, \psi^{\prime}$ lie. Let $G: \psi \rightarrow \psi^{\prime}$ be another open spin foam of this type, possibly with a different underlying $C W$ complex. If the products of $10 j$ symbols over the vertices in $F$ and $G$ are both nonzero, then these products have the same sign.

Proof - Glue $F$ and $G$ together along $\psi$ and $\psi^{\prime}$ to get a closed spin foam $H$. Since the product of $10 j$ symbols for $F$ and $G$ are both nonzero, the same is true of $H$. In fact, the result for $H$ is the product of the results for $F$ and $G$. By Theorem 1 , the sign of the evaluation of $H$ is positive, so the signs of the evaluations of $F$ and $G$ must be the same.

This corollary can be sharpened using an independent argument which we now sketch. This sharpened version says that if the evaluation of $F$ is nonzero, then its sign is $(-1)^{2 J}$, where $J$ is the sum of the spins labelling the edges of $\psi$ and $\psi^{\prime}$. To prove this fact, note that any spin foam is a composite of spin foams with only one vertex, since we can slide a cross-section generically from $\psi$ to $\psi^{\prime}$, so that it passes one vertex at a time. It thus suffices to consider the case where $F$ contains only one vertex. For this, one can examine each of the possible ways in which $\psi$ can differ from $\psi^{\prime}$ when there is only a single vertex in $F$, and note that in each case, the sign of the $10 j$ symbol at that vertex is $(-1)^{2 J}$. For example, a portion of $\psi$ that looks like:

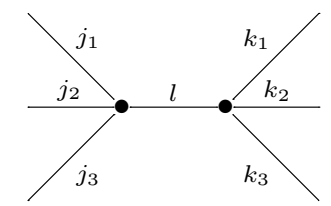

can change to:

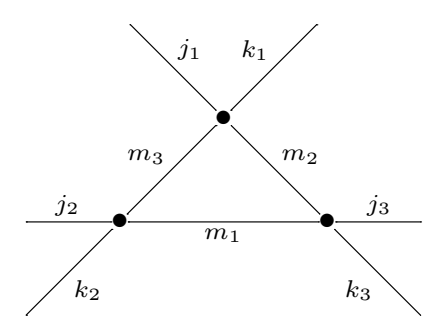

This is the so-called ' $2-3$ Pachner move'. The sum of the ten spins involved in the $10 j$ symbol is $j_{1}+j_{2}+j_{3}+k_{1}+k_{2}+k_{3}+l+m_{1}+m_{2}+m_{3}$. Since the evaluation is nonzero, $(-1)^{2\left(j_{1}+j_{2}+j_{3}\right)},(-1)^{2 l}$ and $(-1)^{2\left(k_{1}+k_{2}+k_{3}\right)}$ are equal. Thus the sign of the $10 j$ symbol is $(-1)^{2\left(l+m_{1}+m_{2}+m_{3}\right)}$. This is equal to $(-1)^{2 J}$, where $J$ is the sum of the spins labelling $\psi$ and $\psi^{\prime}$, since most of the spins appear twice.

There are other transitions that can occur when the cross section passes a vertex, such as the 0-5 and 1-4 Pachner moves. Also, the cross section can pass a cup or a cap in a spin foam edge, which replaces two vertices with one, or vice versa. In all of these cases, one can check that the signs work out as claimed.

\section{LOREnTZiAn MODEL}

The Barrett-Crane model can be defined for any dimension of spacetime and any signature [16]. However, our proof of nonnegativity for spin foam amplitudes in the 
4-dimensional Riemannian Barrett-Crane model relies on two miracles specific to this case: $\operatorname{Spin}(4)$ is a product of two copies of $\mathrm{SU}(2)$, and its simple representations factor as $j \otimes j$. This is what gives the weighted sum of squares in equation (11). These miracles do not occur in the 4-dimensional Lorentzian model, so we are unable to prove nonnegativity in this case. However, we have obtained some numerical evidence for it, which we present here.

As in the Riemannian case, there is some controversy concerning the correct edge and face amplitudes in the Lorentzian Barrett-Crane model. However, in the version for which Crane, Perez and Rovelli [14] have proved convergence of the partition function, these amplitudes are nonnegative. This is also true in all the most obvious variants. Given this, to prove that the amplitude of a spin foam is nonnegative, it suffices to show the vertex amplitudes are nonnegative.

The vertex amplitudes in the Lorentzian Barrett-Crane model are given by the Lorentzian $10 j$ symbols [11]. These are obtained by evaluating $\operatorname{Spin}(3,1)$ spin networks of this form:

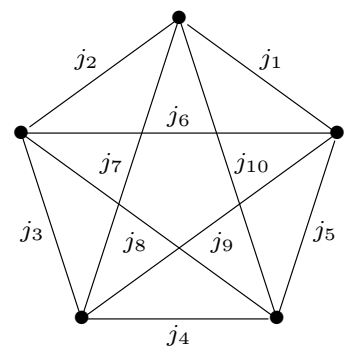

Here the edges are labelled by arbitrary nonnegative real numbers $j_{i}$, which describe simple representations of the group $\operatorname{Spin}(3,1)=\mathrm{SL}(2, \mathbb{C})$. The vertices are all labelled with a special intertwiner called the Lorentzian Barrett-Crane intertwiner. The simple representation $j$ is the space of all solutions to the equation $\nabla^{2} f=-\left(j^{2}+1\right) f$ on 3 dimensional hyperbolic space that have square-integrable boundary data on the sphere at infinity. Since these representations are infinite-dimensional, a careful procedure must be used to evaluate the $10 j$ spin network. The end result is the following integral:

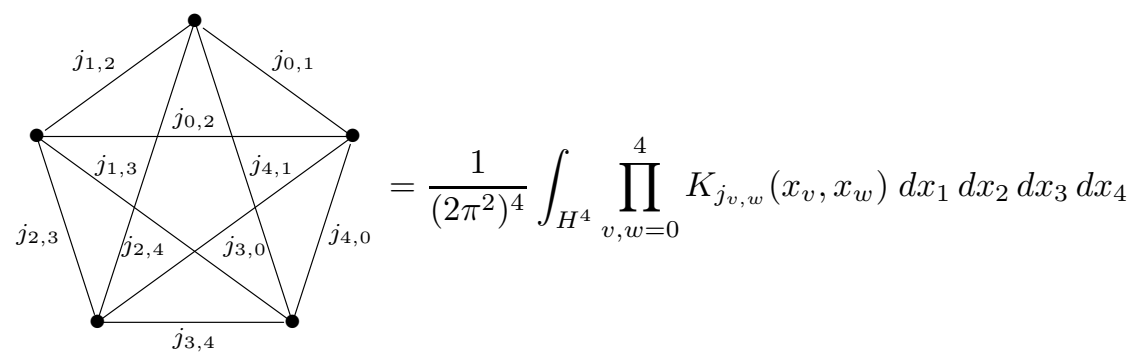

Here $H$ is 3-dimensional hyperbolic space, $d x$ stands for the volume form associated to the Riemannian metric on $H$, and $K_{j}$ is the integral kernel for the projection onto the simple representation $j$, namely

$$
K_{j}(x, y)=\frac{\sin j r}{j \sinh r}
$$

where $r$ is the distance betweeen $x, y \in H$. The answer is independent of the free variable $x_{0}$, which may be held fixed at a convenient point in $H$. While this integral has been shown to converge [7], no 'closed form' is known, and it is difficult to compute numerically because it involves 12 variables and an oscillatory integrand. These difficulties 
become particularly severe as the $j_{i}$ become large, since then the integrand oscillates more rapidly.

We have used two methods to compute this integral numerically for certain choices of $j_{i}$. In both methods we start by writing the integral in hyperbolic spherical coordinates $(r, \theta, \phi) \in[0, \infty) \times[0,2 \pi) \times[0, \pi)$. Then we do a coordinate transformation mapping the radial coordinates $r_{i}$ of the points $x_{1}, x_{2}, x_{3}, x_{4}$ to the interval $[0,1)$ via $r_{i} \mapsto r_{i} /\left(1+r_{i}\right)$. Including the Jacobian of this transformation, the result is an integral with respect to Lebesgue measure on a product of four copies of $[0,1) \times[0,2 \pi) \times[0, \pi)$. Finally, using invariance properties of the integrand, we can reduce this to a 9 -dimensional integral by eliminating the integrals over $\theta_{1}, \phi_{1}$ and $\theta_{2}$.

The first method proceeds by a straightforward Monte Carlo calculation, uniformly sampling the space over which the integral is being taken, and averaging the results. Table 1 lists some results obtained by this method.

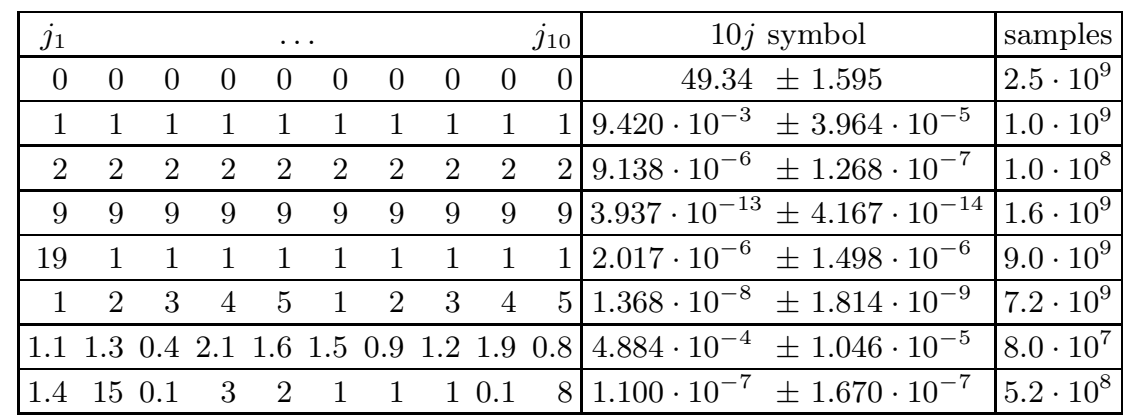

Table 1: Lorentzian $10 j$ symbols — Monte Carlo results

The second method uses the 'Vegas' algorithm. This is an adaptive algorithm that combines importance sampling and stratified sampling. The rather complicated details are explained in Section 7.8 of the book Numerical Recipes [19]. Table 2 lists some results obtained by this method. Each entry in the table represents a weighted average of independent runs of the Vegas algorithm.

\begin{tabular}{|c|c|c|c|c|c|c|c|c|c|c|c|c|c|}
\hline$j_{1}$ & & & & & & & & & & & 10 & $10 j$ symbol & samples \\
\hline 0 & 0 & 0 & & 0 & 0 & 0 & 0 & & 0 & 0 & 0 & $53.42 \pm .0689$ & $5.0 \cdot 10^{8}$ \\
\hline 1 & 1 & 1 & & 1 & 1 & 1 & 1 & & 1 & 1 & 1 & $9.526 \cdot 10^{-3} \pm 1.367 \cdot 10^{-6}$ & $7.5 \cdot 10^{8}$ \\
\hline 2 & 2 & 2 & & 2 & 2 & 2 & 2 & & 2 & 2 & 2 & $9.095 \cdot 10^{-6} \pm 8.153 \cdot 10^{-9}$ & $5.0 \cdot 10^{8}$ \\
\hline 9 & 9 & 9 & & 9 & 9 & 9 & 9 & & 9 & 9 & 9 & $3.626 \cdot 10^{-13} \pm 1.226 \cdot 10^{-15}$ & $1.3 \cdot 10^{9}$ \\
\hline 19 & 1 & 1 & & 1 & 1 & 1 & 1 & & 1 & 1 & 1 & $5.602 \cdot 10^{-8} \pm 6.934 \cdot 10^{-8}$ & $6.5 \cdot 10^{9}$ \\
\hline 1 & 2 & 3 & & 4 & 5 & 1 & 2 & & 3 & 4 & 5 & $1.162 \cdot 10^{-8} \pm 6.057 \cdot 10^{-11}$ & $1.7 \cdot 10^{10}$ \\
\hline 1.1 & 1.3 & 0.4 & 42. & & 1.6 & 1.5 & 0.9 & 1. & & 1.90 & 0.8 & $5.029 \cdot 10^{-4} \pm 9.258 \cdot 10^{-7}$ & $5.0 \cdot 10^{7}$ \\
\hline 1.4 & 15 & 0.1 & & 3 & 2 & 1 & 1 & & 10 & 0.1 & 8 & $3.068 \cdot 10^{-9} \pm 6.860 \cdot 10^{-9}$ & $5.5 \cdot 10^{8}$ \\
\hline
\end{tabular}

Table 2: Lorentzian $10 j$ symbols — Vegas results

In both these tables, the error estimates are calculated as

$$
\Delta f=V \sqrt{\frac{\left\langle f^{2}\right\rangle-\langle f\rangle^{2}}{N}},
$$


where $\langle f\rangle$ denotes the mean of the samples of the function $f, N$ is the number of samples, and $V$ is the volume of the region of integration. In the case of the Vegas algorithm the samples are not uniformly chosen, so the mean is a weighted one, as explained in Numerical Recipes.

The reader can easily note a few things from these tables. First, the computed $10 j$ symbols are always nonnegative. In two cases, namely

$$
\left(j_{1}, \ldots, j_{10}\right)=(19,1,1,1,1,1,1,1,1,1)
$$

and

$$
\left(j_{1}, \ldots, j_{10}\right)=(1.4,15,0.1,3,2,1,1,1,0.1,8)
$$

the computed $10 j$ symbols are smaller than their error bars, consistent with them being zero or even negative. However, this is understandable, since there is a good reason to expect the $10 j$ symbols to be very small in these cases. The reason is that the Barrett-Crane intertwiner

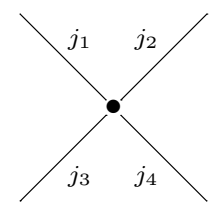

becomes exponentially damped when the spins $j_{1}, \ldots, j_{4}$ leave the region defined by the 'tetrahedron inequalities':

$$
\begin{aligned}
& j_{1} \leq j_{2}+j_{3}+j_{4}, \\
& j_{2} \leq j_{1}+j_{3}+j_{4}, \\
& j_{3} \leq j_{1}+j_{2}+j_{4}, \\
& j_{4} \leq j_{1}+j_{2}+j_{3} .
\end{aligned}
$$

This is most easily seen from the explicit formula in [11] for the value of the $\operatorname{Spin}(4)$ spin network

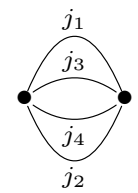

which can be thought of as the norm squared of the Barrett-Crane intertwiner.

Second, the Vegas results generally have smaller error bars than the corresponding Monte Carlo results, and usually lie within the error bars of the Monte Carlo results. This suggests (but does not rigorously prove) that the Vegas algorithm gives more accurate values for the $10 j$ symbols.

Finally, the large sample sizes required to obtain even tolerable accuracy show the difficulty of these numerical computations. It will be important to develop new theoretical methods to prove or disprove the conjectured nonnegativity of these Lorentzian $10 j$ symbols, and also new algorithms to more efficiently compute them.

\section{Conclusions}

Given the results here together with the efficient algorithm for computing the Riemannian $10 j$ symbols described in a companion paper [12], it is now practical to study the Riemannian Barrett-Crane model using the Metropolis algorithm. Some results 
along these lines will appear in another paper [8]. For the Lorentzian model we still need better formulas for the $10 j$ symbols, both for proving nonnegativity and for numerical computations.

The physical significance of Corollary 1 is still somewhat mysterious. Multiplying the usual spin network states by suitable signs, it means we can find orthonormal bases of the kinematical Hilbert spaces of any triangulated compact 3-manifolds $S$ and $S^{\prime}$ such that for any triangulated cobordism $M: S \rightarrow S^{\prime}$, the matrix elements of the operator $Z(M): Z(S) \rightarrow Z\left(S^{\prime}\right)$ are always a sum of nonnegative terms, one for each spin foam $F: \psi \rightarrow \psi^{\prime}$ in the dual 2-skeleton of $M$. At first it was suggested that this means there is no destructive interference between spin foams in the Riemannian Barrett-Crane model. Subsequent discussions have clarified this issue somewhat. Indeed, if a unitary operator has all nonnegative matrix elements in some basis it must be rather trivial, simply acting as a permutation of the basis. However, thanks to the 'problem of time' in quantum gravity, it is more natural to use a sum over spin foams to compute, not a time evolution operator, but the projection from the space spanned by spin networks onto the physical Hilbert space of quantum gravity 4,25$]$. There is no reason for this to be unitary; it should be something more like a positive operator. There is thus no contradiction if it has nonnegative matrix elements. Indeed, this also occurs in other theories, such as the quantum mechanics of a relativistic free particle, and also quantum gravity in $2+1$ dimensions. However, we still lack a general principle explaining why all spin foams $F: \psi \rightarrow \psi^{\prime}$ should have real amplitudes of the same sign.

\section{ACKNOWLEDGEMENTS}

We would like to thank John Barrett, Greg Egan and Tom Halford for conversations and code related to this project. We also thank Lee Smolin for drawing attention to the need for numerical methods suited to real-time path integrals, Rafael Sorkin for raising the issue of a possible lack of destructive interference in models with positive spin foam amplitudes, and Abhay Ashtekar, Alejandro Perez, and Thomas Thiemann for clarifying this issue.

\section{REFERENCES}

[1] J. Ambjørn. J. Jurkiewicz and R. Loll, A non-perturbative Lorentzian path integral for gravity, available as hep-th/0002050.

[2] J. Ambjørn, J. Jurkiewicz and R. Loll, Non-perturbative 3d Lorentzian quantum gravity, Phys. Rev. D64 (2001), 044011.

[3] A. Ashtekar, D. Marolf, J. Mourão and T. Thiemann, Constructing Hamiltonian quantum theories from path integrals in a diffeomorphism invariant context, Class. Quant. Grav. 17 (2000), 49194940.

[4] M. Arnsdorf, Relating covariant and canonical approaches to triangulated models of quantum gravity, available as gr-qc/0110026.

[5] J. C. Baez, Spin foam models, Class. Quantum Grav. 15 (1998), 1827-1858.

[6] J. C. Baez, An introduction to spin foam models of quantum gravity and BF theory, in Geometry and Quantum Physics, eds. H. Gausterer and H. Grosse, Springer Verlag, Berlin, 2000.

[7] J. C. Baez and J. W. Barrett, Integrability for relativistic spin networks, to appear in Class. Quantum Grav., available as gr-qc/0101107.

[8] J. C. Baez, J. D. Christensen. T. R. Halford and D. C. Tsang, Spin foam models of Riemannian quantum gravity, available as gr-qc/0202017.

[9] J. W. Barrett, The classical evaluation of relativistic spin networks, Adv. Theor. Math. Phys. 2 (1998), 593-600.

[10] J. W. Barrett, L. Crane, Relativistic spin networks and quantum gravity, Jour. Math. Phys. 39 (1998), 3296-3302. 
[11] J. W. Barrett and L. Crane, A Lorentzian signature model for quantum general relativity, Class. Quantum Grav. 17 (2000), 3101-3118.

[12] J. D. Christensen and G. Egan, An efficient algorithm for the Riemannian $10 j$ symbols, to appear in Class. Quantum Grav.. Also available as gr-qc/0110045

[13] J. S. Carter, D. E. Flath and M. Saito, The Classical and Quantum 6j-Symbols, Princeton U. Press, Princeton, 1995.

[14] L. Crane, A. Perez and C. Rovelli, A finiteness proof for the Lorentzian state sum spin foam model for quantum general relativity, available as gr-qc/0104057.

[15] R. De Pietri, L. Freidel, K. Krasnov and C. Rovelli, Barrett-Crane model from a Boulatov-Ooguri field theory over a homogeneous space, Nucl. Phys. B574 (2000), 785-806.

[16] L. Freidel, K. Krasnov and R. Puzio, $B F$ description of higher-dimensional gravity theories, Adv. Theor. Math. Phys. 3 (1999), 1289-1324.

[17] L. Kauffman and S. Lins, Temperley-Lieb Recoupling Theory and Invariants of 3-Manifolds, Princeton U. Press, Princeton, New Jersey, 1994.

[18] W. Massey, Singular Homology Theory, Springer Verlag, Berlin, 1980.

[19] W. H. Press, S. A. Teukolsky, W. T. Vetterling and B. P. Flannery, Numerical Recipes in C: The Art of Scientific Computing, 2nd edition, Cambridge U. Press, Cambridge, 1992. Available at http://lib-www.lanl.gov/numerical/index.html.

[20] D. Oriti, Spacetime geometrv from algebra: spin foam models for non-perturbative quantum gravity, available as gr-qc/0106091.

[21] A. Perez, Finiteness of a spin foam model for euclidean quantum general relativity, Nucl. Phys. B599 (2001) 427-434.

[22] A. Perez and C. Rovelli, A spin foam model without bubble divergences, Nucl. Phys. B599 (2001) $255-282$.

[23] M. Reisenberger and C. Rovelli, "Sum over surfaces" form of loop quantum gravity, Phys. Rev. D56 (1997), 3490-3508.

[24] C. Rourke and B. Sanderson, Introduction to Piecewise-Linear Topology, Springer Verlag, Berlin, 1972.

[25] C. Rovelli, The projector on physical states in loop quantum gravity, Phys. Rev. D59 (1999), 104015.

[26] D. N. Yetter, Generalized Barrett-Crane vertices and invariants of embedded graphs, J. Knot Theory Ram. 8 (1999), 815-829.

John C. Baez, Department of Mathematics, University of California, Riverside, CaliforNIA 92521

E-mail address: baez@math.ucr.edu

J. Daniel Christensen, Department of Mathematics, University of Western Ontario, LonDON, ON N6A 5B7 CANADA

E-mail address: jdc@uwo.ca 